\title{
Hemiballismus - Hemichorea: A Case Report
}

\section{Sonmezler A*}

Department of Neurology, Adana City Research and Training Hospital, Turkey

*Corresponding author: Abdurrahman Sonmezler, Department of Neurology, Adana

City Research and Training Hospital, Adana-Turkey, Email: sonmezlera@gmail.com

\section{Case Report}

Volume 4 Issue 2

Received Date: May 31, 2019

Published Date: July 03, 2019

\section{Abstract}

Hemiballismus-hemichorea associated with lesions related to the subthalamic nucleus or its connections. Hemiballismus hemichorea have also been reported in pallidus, caudate core, putamen, thalamus, corona radiata, substantia nigra, premotor and motor cortex lesions. Ballismus is a very large amplitude korean movement that involves the proximal part of the extremities.It is usually unilateral.This condition, which is defined as hemiballismus, is associated with lesions related to the subtalamic nucleus or connections of the contralateral side .Choreic and ballistic movements have been reported in the lesions that cut the afferent and efferent subtalamopallidal pathways of the basal ganglil. In this article we report a 54-year-old woman with hemiballismus-hemichorea who developed after acute ischemia in the globus pallidus.

Keywords: Acute ischemia; Hemiballismus-hemichorea; Globus pallidus

\section{Case Report}

A fifty-four-year-old female patient was admitted with sudden onset,severe, involuntary, wide-amplitude, irregular and continuous movements in the right arm and leg. Her medical history was included hypertension, diabetes mellitus and hyperlipidemia. There was no feature in the family history. In her neurological examination;she had severe ballistic and choreic movements on her left arm and leg. Her routine whole blood and biochemical tests was normal. Electrocardiography (ECG) was in normal sinus rhythm. There was no bleeding in the computed tomography (CT). In brain diffusion magnetic resonance imaging (MRI); acute diffusion restriction consistent with ischemia was observed in the right globus pallidus (video 1). In the examination of the patient for etiology of ischemic stroke; transthoracic echocardiography, colored carotid artery and vertebral artery Doppler ultrasonography were normal. The patient was treated with clopidogrel due to ischemia. Haloperidol and valproic acid treatments were applied primarily for haemiballismus-hemichorea. Clonozepam was added to the treatment because hemiballism was limiting the activities of daily living.
After 3 months complete recovery was observed. All treatments given for hyperkinetic movement disorder were terminated.

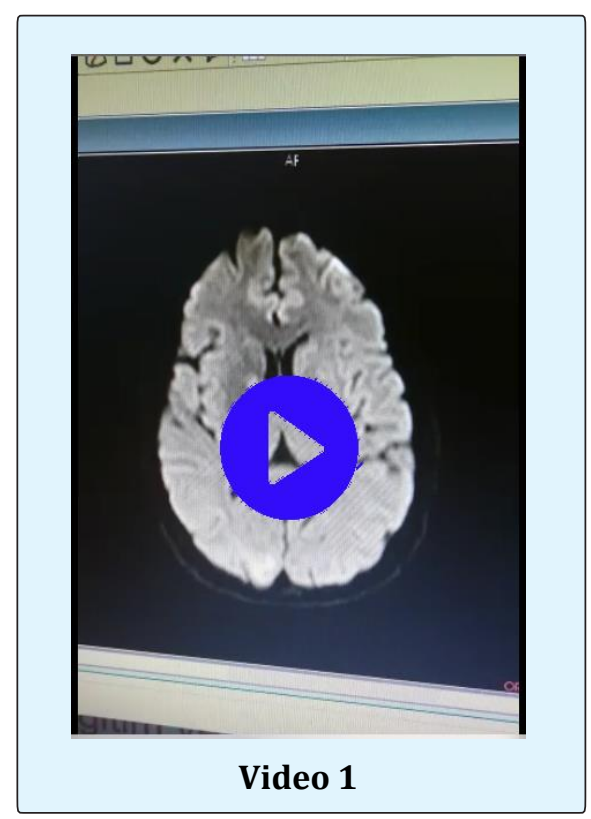




\section{Anaesthesia \& Critical Care Medicine Journal}

Brain diffusion magnetic resonance imaging (MRI); acute diffusion restriction consistent with ischemia was observed in the right globus pallidus

\section{Discussion}

Vascular hemiballism or hemichorea is usually related to an ischemic or hemorrhagic lesion of the basal ganglia and adjacent white matter in the territory of the middle or the posterior cerebral artery [1,2]. Hemichorea consists of continuous random, anarchic and jerking movements involving both the distal and proximal muscles (but is sometimes localized more distally), whereas hemiballismus is characterized by violent irregular flinging movements of the limbs due to contractions of the proximal muscles. The pathogenesis of hemichorea caused by contralateral lesions of the striatal neurons of the indirect striatothalamocortical pathways may be explained by interruption of gamma-aminobutyric acid (GABA) transmission from the striatum to the external globus pallidus (GPE), which can then increase GPE neuronal activity and inhibit the subthalamic nucleus. Such inhibition would induce a loss of control of the internal globus pallidus (GPI) neurons which, in turn, can finally lead to loss of inhibition of the motor thalamus [3]. The classic knowledge, associating the dysfunction of STN with hemiballismus, emphasizes the interruption of excitatory input from the STN to the globus pallidus (GPi) (disrupting the indirect pathway) as the major mechanism of hemiballismus [4]. Laganiere, et al. found that motor pathways implicated in the hemiballismus were considerably complex and widely distributed throughout the brain [5]. Hemichorea-hemiballism syndrome is among the most frequently seen post-stroke involuntary movement disorders [3]. In cases of persistent resistant, deep brain stimulation is applicable. Our patient was treated with haloperidol and valproic acid.Clonozepam was added to the treatment because hemiballism was limiting the activities of daily living. After 3 months complete recovery was observed. All treatments given for hyperkinetic movement disorder were terminated.

\section{References}

1. McCollum D, Silvers S, Dawson SB, Barrett KM (2013) Resolution of acute onset hemichorea-hemiballismus after treatment with intravenous tissue plasminogen activator. Neurohospitalist 3(3): 131-134.

2. Park SY, Kim HJ, Cho YJ, Cho JY, Hong KS (2009) Recurrent hemichorea following a single infarction in the contralateral subthalamic nucleus. Mov Disord 24(4): 617-618.

3. Chung SJ, Im JH, Lee MC, Kim JS(2004) Hemichorea after stroke: clinical-radiological correlation. J Neurol 251(6): 725-729.

4. Laganiere S, Boes AD, Fox MD (2016) Network localization of hemichorea- hemiballismus. Neurology 86(23): 2187-2195.

5. Postuma RB, Lang AE (2003) Hemiballism:Revisiting a classic disorder. Lancet Neurol 2(11): 661-668. 